\title{
Paraneoplastic Cushing Syndrome in Gastrointestinal Neuroendocrine Tumour
}

\author{
Laurent Mineur Rania Boustany Léa Vazquez \\ Institute Sainte Catherine, Gastrointestinal and Liver Cancer Unit, Avignon, France
}

\author{
Keywords \\ Ectopic Cushing syndrome · Neuroendocrine tumours · WHO grade 1 - Paraneoplastic \\ syndromes · Chromogranin A
}

\begin{abstract}
Ectopic production of adrenocorticotropic hormone (ACTH) by gastrointestinal neuroendocrine tumours (NETs) is relatively uncommon. We report a rare case of a liver metastatic G1 low-grade NET of the intestine that induced hypercortisolism after surgical resection. A 50 -year-old man was admitted for an intestinal obstruction caused by a tumour of the intestine. Paraneoplastic Cushing syndrome was diagnosed more than a year later following the appearance of cushingoid symptoms, despite stable disease according to RECIST criteria but chromogranin A increase. Ketoconazole and sandostatin medical treatment and liver chemoembolization never managed to control the hypercortisolism unlike the bilateral adrenalectomy. The identification and effective management of this uncommon statement of ectopic ACTH secretion is important to improve the patient's prognosis and quality of life.
\end{abstract}

\section{Introduction}

Neuroendocrine tumours (NETs) are a relatively rare and heterogeneous tumour type, comprising about $2 \%$ of all malignancies [1]. The gastrointestinal (GI) and pancreatic tract and lungs are the most common primary tumour sites, with $62 \%-67 \%$ and $22 \%-27 \%$, respectively, and within the GI tract, most of them occurs in the small bowel or the appendix [2, 3]. Since 2010 and the latest version of the WHO classification, GI and pancreatic NETs are subdivided 
according to their mitotic count or Ki67 index, associated with cellular proliferation. Welldifferentiated NETs are relatively low-aggressive tumours, with a rather indolent disease course and a good prognosis in most patients. Nevertheless, some NETs with a low-grade histologic appearance may behave aggressively with rapid growth and metastasis proliferation $[4,5]$. Because of this low incidence, tumour heterogeneity, lack of awareness, and nonuniform classifications, GI and pancreatic NETs remain a poorly understood disease, and delayed diagnosis is common among these $[6,7]$.

Paraneoplastic Cushing syndrome (PCS) represents approximately 10\% of all Cushing syndrome and is frequently caused by NETs $[8,9]$. While PCS is common with lung NETs ( $>50 \%$ of PCS), this paraneoplastic syndrome is relatively uncommon associated with GI NETs and only described in isolated case reports. Nevertheless, knowing the indolent course of low-grade NETs and the clinical symptoms of cushingoid appearance resulting from prolonged exposure to excessive glucocorticoids, PCS is typically present before cancer detection [8], and surgery is curative in $>80 \%$ of patients $[10,11]$. For the remaining $20 \%$, effective management is necessary, given the risk of infections and thromboembolic events due to the immunosuppressive effect and the hypercoagulable state [11]. For patients with medically unmanageable hypercortisolism, synchronous bilateral adrenalectomy is an effective and safe treatment [12]. We describe a case of typical metastatic intestinal NETs associated with a late ectopic Cushing syndrome, which was managed with synchronous bilateral adrenalectomy.

\section{Case Presentation}

We describe the case of a 50-year-old man admitted to the emergency department for an intestinal obstruction caused by an intestinal tumour. Anatomopathological analysis of the resected specimen and lymph nodes revealed an NET. Three nodes out of 12 removed were positive for cancer localization. The tumour presented serosa infiltration and perineural, vascular and lymphatics vessel invasion. The primary location could not be confirmed histologically between the ileum and appendix. Our diagnosis was pT3N1 according to the American Joint Committee on Cancer (AJCC) classification. An immunohistochemistry analysis revealed a Ki-67 expression $<2 \%$. Mitotic count/ 10 was $2 \times 10$ high-power fields, and cells showed well differentiation. So, according to the WHO classification, this tumour was classified as G1 NET. ${ }^{111}$ In-Octreoscan (Octreoscan) revealed lymph node and multifocal liver metastases.

After discussion with a multidisciplinary team, the patient was started treatment with somatostatin analogue. Twelve months later, although computerized tomography (CT) scan showed stable disease, patient physical examination revealed facial puffiness with fatty tissue deposits in the face, generalized oedema, muscle weakness, and wasting. He also reports polydipsia, insomnia, and balance disorders. We noted however a discreet increase in the chromogranin A (CgA) value, from $55 \mathrm{ng} / \mathrm{mL}$ to $199 \mathrm{ng} / \mathrm{mL}$ (with a diagnostic value of 1,700 ng/ $\mathrm{mL}$ ) without an increase in the urinary 5-HIAA level.

Laboratory tests revealed an 8.00 a.m. cortisol level of $888 \mathrm{nmol} / \mathrm{L}$, an adrenocorticotropic hormone (ACTH) level of $96.5 \mathrm{pg} / \mathrm{mL}$, and 24-h urine free cortisol of 1,494 $\mu \mathrm{g}$. A highdose dexamethasone suppression test showed no cortisol suppression. The patient was diagnosed with ACTH-dependent Cushing syndrome. Magnetic resonance imaging (MRI) of the brain showed a normal pituitary gland, confirming the PCS diagnosis. Ketoconazole treatment associated with sandostatin alleviated hypercortisolism within a month, with a cortisol level within normal laboratory ranges. Two months later, secondary diabetes mellitus was discovered and managed effectively with insulin glargine.

Four months later and despite stable disease according to RECIST criteria, cortisol levels increased considerably, with cortisol values similar to diagnosis without ketoconazole increased 


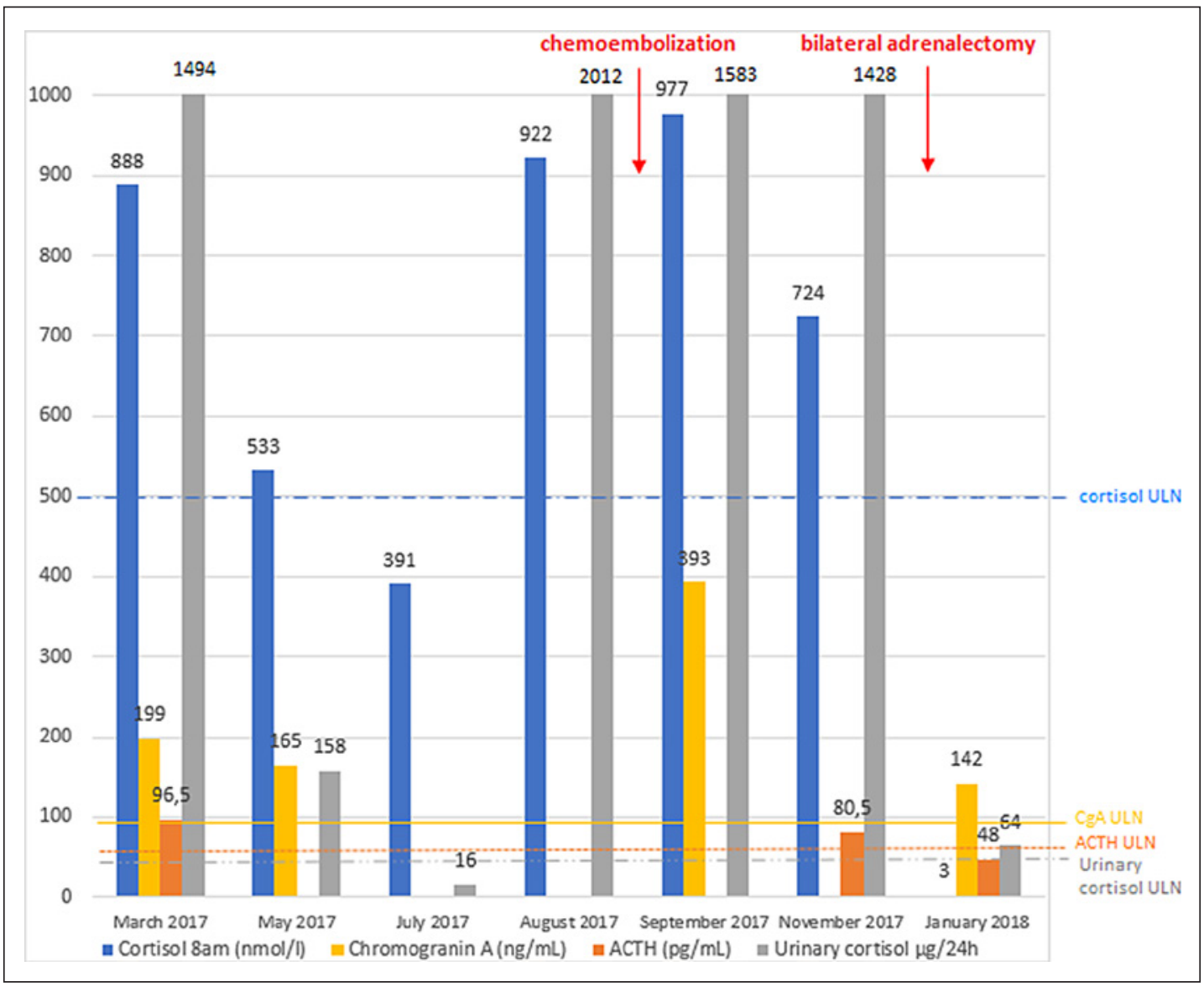

Fig. 1. Histogram of 8:00 a.m. plasmatic cortisol, ACTH, 24-h urinary cortisol, and CgA levels from Cushing syndrome diagnosis to bilateral adrenalectomy. ACTH, adrenocorticotropic-hormone; ULN, upper limit of normal; 8:00 a.m. cortisol normal ranges (172-497) nmol/L; ACTH normal ranges (7-63) ng/L; 24-h urinary cortisol normal ranges (20-50) $\mu \mathrm{g} / 24 \mathrm{~h}$; CgA normal ranges (27-94) ng/mL. ACTH, adrenocorticotropic hormone; $\mathrm{CgA}$, chromogranin $\mathrm{A}$.

response. Moreover, diabetes became complicated to manage. Also we noted an increase in CgA value, from $165 \mathrm{ng} / \mathrm{mL}$ to $393 \mathrm{ng} / \mathrm{mL}$. Chemoembolization was performed on liver metastases without any effectiveness on hypercortisolism. Adding targeted therapy with mTOR inhibitor (everolimus) was considered. Nevertheless, given the magnitude of drug interaction, the use of everolimus should be avoided in ketoconazole-treated patients, or vice versa.

Considering the risks for the patient and expected benefits, synchronous bilateral adrenalectomy was performed. It resolved hypercortisolism and permitted to stabilize diabetes (shown in Fig. 1). Everolimus treatment has been started 1 month after the surgery. Twelve months after everolimus initiation, the patient CT scan still showed stable disease, according to RECIST criteria and a stable CgA value.

\section{Discussion}

Approximately $10 \%$ of Cushing syndrome is paraneoplastic and may result in many tumours, preferentially lung cancer (50-60\% of time), with $1-2 \%$ of lung NET and about $5 \%$ of small-cell lung cancer associated with Cushing syndrome $[8,11,13]$. Others reported sites 
of malignancy include the thymus, thyroid, pancreas, and adrenals. Except for the pancreas, PCS secondary to GI NET (appendix, duodenum, ileum, colon, and anal canal) is extremely rare, and only isolated case reports have described this syndrome.

In paraneoplastic endocrine syndrome cases, symptoms are due to secretion of hormones by malignant cells or secondary to the impact of neoplastic cell antibodies on normal cells. PCS arises from tumour secretion of ACTH or CRH, resulting in production and release of cortisol from the adrenal glands. Unlike paraneoplastic endocrine syndromes that present most of the time after cancer diagnosis, PCS typically appears before cancer detection and similarly relapse may herald tumour recurrence $[11,14]$. In our case, no symptoms related to hypercortisolaemia led the patient to consult before obstructive syndrome. The occurrence of hypercortisolaemia 12 months after diagnosis was not linked to imaging progression according to RECIST criteria. However, concurrent CgA increase should be noted.

Commonly measured tumour markers in NETs include serum CgA and 5-HIAA, the final secreted product of serotonin, levels in a 24-h urine sample. Elevated levels of circulating of CgA have been associated with almost all types of NETs, including those arising from GI tract but also pheochromocytomas [15]. The clinical sensitivity of CgA has been demonstrated to depend on the threshold cut-off, on NET primary location, and on the spread of the disease, especially the existence of liver metastases [16]. Indeed, a higher sensitivity was found in patients with midgut NETs and liver metastases, as in our patient. Moreover, with our cut-off level $(94 \mathrm{ng} / \mathrm{mL})$ approximately the same as used in 2 studies $[16,17]$, sensitivity was $62 \%-67 \%$ and specificity was $96 \%$. Furthermore, Korse et al. [18]. postulated that serum CgA was superior to urinary 5-HIAA concerning the prognostic relevance in the follow-up of metastatic midgut NETs. These data are consistent with our patient outcomes for which 5-HIAA was not increased unlike CgA. However, although CgA is currently the best available tumour marker indicating tumour recurrence [19], there are many comorbidities and drugs that may increase $\mathrm{CgA}$ levels and lead to false-positive results. As a result, it is questionable whether the CgA increase in our patient was not rather secondary to cardiovascular or GI disorders, inflammatory diseases, diabetes, or even food intake before CgA measurement [16, 20, 21]. Similarly, many drugs, foods, natural stimulants, and comorbidities may alter the level of 24-h urinary 5-HIAA, positively or negatively.

Cushing syndrome is due to hypercortisolism. Two-thirds of endogenous elevated cortisol is caused by ACTH-secreting pituitary tumours, $15 \%$ by primary adrenal glands and $15 \%$ by ectopic PCS [22]. The first step is laboratory tests with cortisol and ACTH levels to differentiate ACTH-dependent or ACTH-independent Cushing syndrome. When ACTH-dependent Cushing syndrome is confirmed, differentiation between PCS and Cushing disease can be difficult. The high-dose dexamethasone suppression tests help distinguish Cushing disease from PCS, as in our presented case. Indeed, no decrease in blood cortisol during the high-dose test and high ACTH levels are consistent with PCS. Nevertheless, $21-26 \%$ of ectopic ACTH secretions have a positive suppression, about one-third of MRI scans for pituitary adenoma exclusion are false-negative, and occult ectopic ACTH-secreting tumours have been described in about $15 \%$ of adult patients [23-26]. In our patient, both MRI and high-dose dexamethasone suppression test are consistent with PCS. The gold standard diagnosis - inferior petrosal sinus sampling - that demonstrates gradient in ACTH concentration between the affected side sinus and the periphery in pituitary lesions, whereas the absence of this gradient in PCS was not performed because of its invasiveness and its neurological accident risks [27]. Note however although the ACTH level at diagnosis suggests ACTH-dependent Cushing syndrome, the occurrence of adrenal metastasis few months after the diagnosis and explaining the sudden deregulation could be possible and consistent with the $\mathrm{CgA}$ increase but refuted by adrenal gland histology.

\section{Karger'}


Clinical features of PCS depend on the source of production and rate of ACTH synthesis. Characteristically, these patients have severe hypercortisolaemia, leading to low serum potassium levels, diabetes, generalized infections, hypertension, and psychosis. To confirm whether rapidly growing tumours produce sudden onset of symptoms, gradual physical signs are noticed in slower growing tumours [28], as for our patient for whom we suppose that liver metastases started to produce ACTH ectopically. An option for non-resectable neuroendocrine liver lesions, given that the majority of them are hypervascular, is hepatic directed procedures, which include ablative therapy, transarterial embolization, transarterial chemoembolization, and selective internal radiation therapy with yttrium-90 microspheres [29]. Hepatic artery chemoembolization for the treatment of liver metastases from NETs is useful for tumour size reduction and symptom palliation and can be associated with prolonged survival [30]. Nevertheless, chemoembolization on NET liver metastasis-producing ACTH is not well documented. Given the fact that hepatic metastasis chemoembolization was ineffective on hypercortisolism and despite Octreoscan results, there is still a small chance that he harbours somewhere else metastasis-producing ACTH. Indeed, PET-CT imaging with ${ }^{68}$ Gallium-DOTATATE has recently replaced Octreoscan as the new gold standard with a higher detection rate in GI NETs [31].

Hypercortisolism requires a prompt therapeutic management to reduce the risk of development of a potentially fatal emergency. Synchronous bilateral adrenalectomy is an effective and safe treatment for patient with unmanageable ACTH-dependent hypercortisolism [12]. Taking account of the risks to the patient and the lack of effective medical therapeutic possibilities, we have chosen to perform this surgery.

According to the recent consensus guidelines for digestive NETs of the jejunum and ileum, the 5 -year survival rate is $36 \%$ in patients with distant metastases [32]. Several analyses suggest a significant survival benefit in patients who received surgery for the primary tumour even in the presence of metastasis [33]. Moreover, the impact of liver resection or liver-directed therapies on the survival of patients with liver metastasis is unclear with conflicting results [33]. PCS can cause a poor clinical outcome due to various complications with an increase in susceptibility to infection and GI ulceration. Indeed, for small-cell lung cancer and gynaecological malignancies, PCS is associated with accelerated decompensation and poorer response to chemotherapy (Mitchell et al. [14]). Whether these findings can be extrapolated to other malignancies is unknown. However, an early diagnosis and a prompt management can improve patient outcomes through earlier cancer diagnosis or relapse and thus earlier administration of treatment, as was the case with our patient.

\section{Conclusion}

We report an uncommon case with PCS due to a GI NET. The identification of this rare cause of ectopic ACTH secretion can be challenging, but aggressive management is critical to prevent or decelerate the acute decompensation of cancer patients and prolong overall survival. In this context, synchronous bilateral adrenalectomy may be the unique answer.

\section{Statement of Ethics}

Written informed consent was obtained from the patient for publication of this case report and any accompanying images. A copy of the written consent is available for review by the editor-in-chief of this journal.

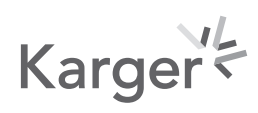




\section{Conflict of interest Statement}

The authors have no conflict of interest to declare.

\section{Funding Sources}

No funding was received for this study.

\section{Author Contributions}

L.M. conceived the study and participated in data collection. L.V. performed the literature search and wrote the manuscript. L.M. and R.B. critically revised the manuscript for important intellectual content. All authors read and approved the final manuscript version.

\section{Data Availability Statement}

The datasets used and analysed during the current study are available from the corresponding author on reasonable request.

\section{References}

1 Basu B, Sirohi B, Corrie P. Systemic therapy for neuroendocrine tumours of gastroenteropancreatic origin. Endocr Relat Cancer. 2010;17(1):R75-90.

2 Taal BG, Visser O. Epidemiology of neuroendocrine tumours. Neuroendocrinol. 2004;80(Suppl 1):3-7.

3 Singer J, Werner F, Koch CA, Bartels M, Aigner T, Lincke T, et al. Ectopic Cushing's syndrome caused by a well differentiated ACTH-secreting neuroendocrine carcinoma of the ileum. Exp Clin Endocrinol Diabetes. 2010; 118:524-9.

4 Farell JM, Pang JC, Kim GE, Tabatabai ZL. Pancreatic neuroendocrine tumors: accurate grading with Ki67 index on fine-needle aspiration specimens using the WHO 2010/ENETS criteria. Cancer Cytopathol. 2014;122(10):770-8.

5 Celotti A, Pulcini G, Schieppati M, Ministrini S, Berruti A, Ronconi M. An unusual case of a well-differentiated neuroendocrine tumour of the ileum with peritoneal carcinomatosis: a case report. World J Surg Oncol. 2015; 13:169.

6 Modlin IM, Oberg K, Chung DC, Jensen RT, de Herder WW, Thakker RV, et al. Gastroenteropancreatic neuroendocrine tumours. Lancet Oncol. 2008;9:61-72.

7 Huang PY, Tsai KL, Liang CM, Tai WC, Rau KM, Wu KL, et al. Prognostic factors of patients with gastroenteropancreatic neuroendocrine neoplasms. Kaohsiung J Med Sci. 2018;34(11):650-6.

8 Pelosof LC, Gerber DE. Paraneoplastic syndromes: an approach to diagnosis and treatment. Mayo Clin Proc. 2010;85(9):838-54.

9 Belissant Benesty O, Nataf V, Ohnona J, Michaud L, Zhang-Yin J, Bertherat J, et al. 68Ga-DOTATOC PET/CT in detecting neuroendocrine tumours responsible for initial or recurrent paraneoplastic Cushing's syndrome. Endocrine. 2019;67(3):708.

10 Escalante A, Guillén Ponce C, Macià Escalante S, Pons Sanz V, Carrato Mena A. Cushing's paraneoplastic syndrome as first manifestation of an adenocarcinoma of unknow origin. Clin Transl Oncol. 2016;8:621-3.

11 Richa CG, Saad KJ, Halabi GH, Gharios EM, Nasr FL, Merheb MT. Case-series of paraneoplastic cushing syndrome in small-cell lung cancer. Endocrinol Diabetes Metab Case Rep. 2018.

12 Chiloiro S, Giampietro A, Raffaelli M, D’Amato G, Bima C, Lauretti L, et al. Synchronous bilateral adrenalectomy in ACTH-dependent hypercortisolism: predictors, biomarkers and outcomes. Endocrine. 2019;66:642-9.

13 Fazel P, Ganesa P, Mennel RG, Austin NA. The ectopic adrenocorticotropic hormone syndrome in carcinoid tumors. Proc. 2008;21:140-3.

14 Mitchell E, Ciccone M, Zhang B, Tsai A, Brunette LL, et al. Paraneoplastic Cushing' s syndrome and hypercalcemia arising from metastatic endometrioid endometrial adenocarcinoma: a case report. Gynecol Oncol Rep. 2019;29:58-60.

15 Zatelli MC, Torta M, Leon A, Ambrosio MR, Gion M, Tomassetti P, et al. Chromogranin A as a marker of neuroendocrine neoplasia: an Italian Multicenter study. Endocr Relat Cancer. 2007;14:473-82. 


\section{Case Reports in Oncology}

\begin{tabular}{l|l}
\hline Case Rep Oncol 2021;14:1407-1413 \\
\hline DOI: 10.1159/000518316 & $\begin{array}{l}\text { @ 2021 The Author(s). Published by S. Karger AG, Basel } \\
\text { www.karger.com/cro }\end{array}$ \\
\hline
\end{tabular}

Mineur et al.: Paraneoplastic Cushing Syndrome in Gastrointestinal Neuroendocrine Tumour

16 Hellman P, Linder F, Hennings J, Hessman O, Eriksson B, Orlefors H, et al. Bilateral adrenalectomy for ectopic Cushing's syndrome-discussions on technique and indication. World J Surg. 2006;30:909-16.

17 Stridsberg M, Eriksson B, Oberg K, Janson ET. A comparison between three commercial kits for chromogranin A measurements. J Endocrinol. 2003;177:337-41.

18 Korse CM, Bonfrer JM, Aaronson NK, Hart AA, Taal BG. Chromogranin A as an alternative to 5-hydroxyindoleacetic acid in the evaluation of symptoms during treatment of patients with neuroendocrine tumors. Neuroendocrinol. 2009;89:296-301.

19 Welin S, Stridsberg M, Cunningham J, Granberg D, Skogseid B, Oberg K, et al. Elevated plasma chromogranin $\mathrm{A}$ is the first indication of recurrence in radically operated midgut carcinoid tumors. Neuroendocrinol. 2009; 89:302-7.

20 Marotta V, Zatelli MC, Sciammarella C, Ambrosio MR, Bondanelli M, Colao A, et al. Chomogranin A as circulating marker for diagnosis and management of neuroendocrine neoplasms: more flaws than fame. Endocr Relat Canc. 2018;25:R11-R29.

21 Broedbaek K, Hilsted L. Chromogranin A as biomarker in diabetes. Biomark Med. 2016;10(11):1181-9.

22 Clotman K, Twickler M, Dirinck E, Den Brande JV, Lammens M, Verhaegen A, et al. Case report an endocrine picture in disguise: a progressive olfactory neuroblastoma complicated with ectopic cushing syndrome. AACE Clin Case Rep. 2017;3:278-83.

23 Tabarin A, Valli N, Chanson P, Bachelot Y, Rohmer V, Bex-Bachellerie V, et al. Usefulness of somatostatin receptor scintigraphy in patients with occult ectopic adrenocorticotropin syndrome. J Clin Endocrinol Metab. 1999;84(4):1193-202.

24 Isidori AM, Kaltsas GA, Pozza C, Frajese V, Newell-Price J, Reznek RH, et al. The ectopic adrenocorticotropin syndrome: clinical features, diagnosis, management, and long-term follow-up. J Clin Endocrinol Metab. 2006; 91(2):371-7.

25 Ilias I, Torpy DJ, Pacak K, Mullen N, Wesley RA, Nieman LK, et al. Cushing's syndrome due to ectopic corticotropin secretion: twenty years' experience at the national institutes of health. J Clin Endocrinol Metab. 2005; 90:4955-62.

26 Philippon M, Castinettia F, Bruea T. Cushing's disease with negative imaging in adults. Ann Endocrinol. 2013; 74(Suppl 1):S23-32.

27 Kenchaiah M, Heyer S. Case report Cushing's syndrome due to ectopic ACTH from bronchial carcinoid: a case report and review. Case Rep Endocrinol. 2012;2012:215038.

28 Paun DL, Vija L, Stan E, Banica A, Bobeica E, Terzea D, et al. Cushing syndrome secondary to ectopic adrenocorticotropic hormone secretion from a Meckel diverticulum neuroendocrine tumor: case report. BMC Endocr Disord. 2015;15:72.

29 Oronsky B, Ma PC, Morgensztern D, Carter CA. Nothing but NET: a review of neuroendocrine tumors and carcinomas. Neoplasia. 2017;19(12):991-1002.

30 Dong XD, Carr BI. Hepatic artery chemoembolization for the treatment of liver metastases from neuroendocrine tumors: a long-term follow-up in 123 patients. Med Oncol. 2011 Dec;28(Suppl 1):S286-90.

31 Wang R, Zheng-Pywell R, Chen HA, Bibb JA, Chen H, Rose JB. Management of gastrointestinal neuroendocrine tumors. Clin Med Insights Endocrinol Diabetes. 2019;12:1179551419884058.

32 Eriksson B, Klöppel G, Krenning E, Ahlman H, Plöckinger U, Wiedenmann B, et al. Consensus guidelines for the management of patients with digestive neuroendocrine tumors well-differentiated jejunal-ileal tumor/ carcinoma. Neuroendocrinol. 2008;87(1):8-19.

33 Shah CP, Mramba LK, Bishnoi R, Unnikrishnan A, Duff JM, Chandana SR, et al. Survival trends of metastatic small intestinal neuroendocrine tumor: a population-based analysid of SEER database. J Gastrointest Oncol. 2019;10(5):869-77. 